\title{
Sleep duration and chronic kidney disease: The Korean Genome and Epidemiology Study (KoGES)-Kangwha study
}

Hansol Choi ${ }^{1,2}$, Hyeon Chang Kim ${ }^{2,3,4}$, Joo Young Lee ${ }^{3}$, Ju-Mi Lee ${ }^{3,4}$, Dong Phil Choi ${ }^{1,2}$, and Il Suh ${ }^{3}$

\author{
${ }^{1}$ Department of Public Health, \\ Yonsei University Graduate \\ School, Seoul; ${ }^{2}$ Cardiovascular and \\ Metabolic Disease Etiology Research \\ Center, ${ }^{3}$ Department of Preventive \\ Medicine, Yonsei University \\ College of Medicine, Seoul, Korea; \\ ${ }^{4}$ Department of Preventive Medicine, \\ Northwestern University Feinberg \\ School of Medicine, Chicago, IL, \\ USA
}

Received: December 24, 2015

Revised : May 13, 2016

Accepted: May 15, 2016

\section{Correspondence to}

Hyeon Chang Kim, M.D.

Department of Preventive

Medicine, Yonsei University

College of Medicine, 50-1

Yonsei-ro, Seodaemun-gu, Seoul

03722, Korea

Tel: +82-2-2228-1883

Fax: +82-2-392-8133

E-mail: hckim@yuhs.ac
Background/Aims: Sleep duration affects health in various ways. The objective of this study was to investigate the associations of sleep duration with chronic kidney disease (CKD) in a Korean adult population.

Methods: This cross-sectional analysis was conducted for total of 1,360 participants who completed baseline health examinations for the Korean Genome and Epidemiology Study-Kangwha study in 2010 to 2011. Sleep habits were measured by an interviewer-assisted questionnaire. Sleep duration was calculated based on the number of hours per day participants had slept over the past 1 year. CKD was defined as either proteinuria or estimated glomerular filtration rate (eGFR) $<60$ $\mathrm{mL} / \mathrm{min} / 1.73 \mathrm{~m}^{2}$. Multiple logistic regression models were applied to examine associations between sleep duration and CKD.

Results: Women with very long sleep duration ( $\geq 9$ hours/day) were at significantly increased odds for having high serum creatinine (odds ratio [OR], 2.936; 95\% confidence interval [CI], 1.176 to 7.326), low eGFR (OR, 3.320; 95\% CI, 1.372 to 8.034), and CKD (OR, 3.112; 95\% CI, 1.315 to 7.363), compared those with a typical sleep duration ( 7 to $<8$ hours/day), after adjusting for sociodemographic status, socioeconomic status, health behaviors, comorbidities, and sleep quality. Among women, for every 1 hour increase in sleep duration per day, there was a $24.6 \%$ increase in the presence of CKD (OR, 1.246; 95\% CI, 1.019 to 1.523). However, among men, sleep duration was not significantly associated with CKD.

Conclusions: Very long sleep duration was independently associated with a higher prevalence of CKD among Korean women. Gender may influence this association.

Keywords: Sleep duration; Sleep quantity; Renal insufficiency, chronic; Kidney function; General population

\section{INTRODUCTION}

Epidemiologic studies suggest that sleep duration may adversely affect one's health in a number of ways. Interestingly, both short and long sleep durations have been shown to be associated with increased risks of all-cause 
mortality, cardiovascular disease, and other chronic diseases [1-3]. Meanwhile, sleeping habits in the general population reflect a complexity of interactions related to the presence of comorbid medical conditions and physical, psychological, social, and lifestyle factors.

Chronic kidney disease (CKD) has emerged as a public health problem worldwide, with reports of increasing prevalence and adverse complications [4-7]. Moreover, CKD has been recognized as a predictor of end-stage kidney disease and cardiometabolic disease [4]. Nevertheless, adverse outcomes of CKD can be prevented through early detection, education, treatment, and intervention to modify harmful lifestyle factors $[8,9]$. In particular, changing one's sleep duration may be effective in preventing CKD. Although cross-sectional and prospective epidemiologic studies have suggested that sleep quality is a significant risk factor for CKD $[10,11]$, little is known of the association between sleep quantity and CKD $[8,12,13]$. While a few studies have suggested that sleep duration can influence kidney function, distinct associations remain unclear. Therefore, we aimed to investigate the relationships between sleep duration and CKD in a Korean adult population.

\section{METHODS}

\section{Study population}

Data for the present study were derived from the Korean Genome and Epidemiology Study (KoGES)-Kangwha study, an ongoing rural community-based prospective cohort. During the baseline survey period from 2006 to 2011, the KoGES-Kangwha study enrolled 4,899 community dwellers from Kangwha Island, Incheon, South Korea. Current cross-sectional analysis was conducted for a total of 1,360 participants who completed the questionnaire concerning sleep (544 men and 816 women aged 36 to 88 years) in 2010 to 2011 . All participants provided written informed consent, and the Institutional Review Board of Severance Hospital, Yonsei University College of Medicine, approved the study protocol.

\section{Measurements}

Trained interviewers obtained information on the participants' demographic characteristics, socioeconomic status (household income, marital status, and working status), previously known diseases, medication use, and health behaviors (sleep, cigarette smoking, alcohol intake, and physical activity) using standardized questionnaires. Depressive symptoms were assessed using the Beck Depression Inventory (BDI), and depression was determined on the basis of BDI score of $\geq 16$ or a self-reported physician's diagnosis [14]. Smoking status, alcohol consumption, and physical activity were categorized as either current or non-current.

Sleep habits were measured by an interviewer-assisted questionnaire. Participants were asked to report the number of hours per day they had slept over the past 1 year, including naps, whether they had trouble falling asleep, whether they had trouble getting back to sleep after they had awaken, snoring behaviors, and the presence of sleep apnea. In the present study, average sleep durations were defined as the total time in bed per day (calculated from bedtime, rise time, and naps). Participants were divided into five groups based upon their reported sleep duration: $<6$ hours/day, "very short sleepers"; 6 to $<7$ hours/day, "short sleepers"; 7 to $<8$ hours/ day, "usual sleepers (reference group)"; 8 to $<9$ hours/ day, "long sleepers"; and $\geq 9$ hours/day, "very long sleepers."

All participants completed health examinations, including anthropometric measurements, blood pressure measurements, blood laboratory tests, bone mineral density measurements, and electrocardiography. Body weight was measured to the nearest $0.1 \mathrm{~kg}$ on a digital scale (GL-600oo-20, CAS Korea, Seoul, Korea), and standing height was measured to the nearest $0.1 \mathrm{~cm}$ on a stadiometer (SECA 225, SECA, Hamburg, Germany). Body mass index (BMI) was calculated as body weight (kg) divided by standing height $\left(\mathrm{m}^{2}\right)$. Resting blood pressures were measured twice at a 5-minute interval using an automatic sphygmomanometer (Dinamap 1846 SX/P, GE Healthcare, Waukesha, WI, USA). Additional measurements were performed, if the first and second measurements differed by $\geq 10 \mathrm{mmHg}$ for either systolic or diastolic blood pressure, and the average of the last two measurements was used for analysis.

Fasting blood samples were collected from the antecubital vein after at least an 8-hour fast. Blood samples were sent to an independent research laboratory center for analysis. Serum creatinine concentrations and blood urea nitrogen concentrations were measured by the col- 
orimetric methods, and serum concentrations of cholesterol were measured by enzymatic methods with an automatic analyzer (ADVIA 1650, Siemens, Tarrytown, NY, USA in 2010; ADVIA 1800, Siemens in 2011). Diabetes mellitus was determined on the basis of fasting glucose concentration ( $\geq 126 \mathrm{mg} / \mathrm{dL}$ ) or a self-reported physician's diagnosis [15]. Hypercholesterolemia was defined (according to the Korean Society of Lipidology and Atherosclerosis criteria) as a total cholesterol concentration $\geq 230 \mathrm{mg} / \mathrm{dL}$ [16], taking a lipid-lowering drug, or a self-reported physician's diagnosis.

Kidney function was estimated by serum creatinine concentration, presence of proteinuria, and estimated glomerular filtration rate (eGFR). Serum creatinine concentration cutoffs were sex specific, corresponding to the study design, with cutoffs of $>1.3 \mathrm{mg} / \mathrm{dL}$ for men and $>1.0 \mathrm{mg} / \mathrm{dL}$ for women. The intra-assay coefficients of variation were $1.7 \%$ at $1.8 \mathrm{mg} / \mathrm{dL}$ and $1.3 \%$ at $8.4 \mathrm{mg} / \mathrm{dL}$. The total coefficients of variation were $3.8 \%$ at $1.8 \mathrm{mg} /$ $\mathrm{dL}$ and $3.7 \%$ at $8.4 \mathrm{mg} / \mathrm{dL}$. Proteinuria was diagnosed as 1+ or more by semiquantitative dipstick test. An eGFR was calculated using the modification of diet in renal disease study equation [17] as follows: $186 \times$ (creatinine $/ 88.4)^{-1.154} \times(\text { age })^{-0.203} \times(0.742$ if female $)$. The diagnosis of CKD was defined as either kidney damage or an eGFR of $<60 \mathrm{~mL} / \mathrm{min} / 1.73 \mathrm{~m}^{2}$, according to criteria set by the National Kidney Foundation Kidney Disease Outcomes Quality Initiative [18].

\section{Statistical analysis}

The distribution of continuous variables was described as means with standard deviations and compared using one-way analysis of variance. Categorical variables were reported as observed numbers and percentages, and compared using the chi-square test. A general linear model using contrast coefficients was used for linear trend analysis of continuous variables, and Cochran-Armitage test and Mantel-Haenszel test were used for categorical variables. Multiple linear regression models were used to assess the independent association between sleep duration and CKD. Sex, age, BMI, systolic blood pressure, smoking status, alcohol consumption, physical activity, diabetes mellitus, hypercholesterolemia, depression, history of cardiovascular disease, history of cancer, menopausal status, socioeconomic status (household income, marital status, and working status), and sleep quality (sleep difficulty, sleep awakeness, snoring, and sleep apnea) were considered as covariates in the model. In addition, multiple logistic regression models were used to estimate odds ratios (OR) with 95\% confidence intervals (CI) for CKD criteria. We performed statistical analyses on the total population, as well as men and women separately, because we found significant sex differences in kidney function. We plotted serum creatinine concentration and eGFR versus average sleep duration expressed on a continuous scale using cubic spline models. In a sensitivity analysis, we calculated age-specific sex-stratified ORs for CKD across the average sleep duration. An additional multiple logistic regression analysis was conducted excluding participants with hypertension $(n=516)$, diabetes mellitus $(n=165)$, and a history of cardiovascular disease $(n=103)$. All statistical analyses were performed using SAS software version 9.2 (SAS, Cary, NC, USA), and statistical significance was defined as a two-sided $p$ value of less than 0.05 .

\section{RESULTS}

The baseline characteristics of all participants are presented in Table 1. Of 1,360 participants, there were 130 very short sleepers ( $<6$ hours/day, 9.6\%), 301 short sleepers ( 6 to $<7$ hours/day, 22.1\%), 458 usual sleepers (7 to $<8$ hours/day, 33.7\%), 319 long sleepers ( 8 to $<9$ hours/ day, 23.5\%), and 152 very long sleepers $(\geq 9$ hours/day, $11.2 \%)$. Among men, the percentages of participants who slept $<6$ and $\geq 9$ hours were $8.6 \%$ and $12.1 \%$, respectively. Among women, the corresponding percentages were $10.2 \%$ and $10.5 \%$, respectively. The number of participants with a very short sleep duration was greater for women than for men, and the number of participants with a very long sleep duration was smaller for women than for men. The mean sleep duration was 431.4 minutes/day for men and 430.6 minutes/day for women. The mean serum creatinine concentration was $1.1 \mathrm{mg} / \mathrm{dL}$ for men and $0.9 \mathrm{mg} / \mathrm{dL}$ for women, and was thus significantly higher among men. The prevalence of high serum creatinine concentration was 7.4\% among both men and women. The mean eGFR was $76.0 \mathrm{~mL} / \mathrm{min} / 1.73 \mathrm{~m}^{2}$ for men and $73.7 \mathrm{~mL} / \mathrm{min} / 1.73 \mathrm{~m}^{2}$ for women, and was thus significantly higher among 
Table 1. Baseline characteristics of the study population $(n=1,360)$

\begin{tabular}{|c|c|c|c|c|}
\hline Variable & Total $(\mathrm{n}=1,360)$ & $\operatorname{Men}(n=544)$ & Women $(n=816)$ & $p$ value \\
\hline Age, yr & $60.0 \pm 10.3$ & $61.5 \pm 10.2$ & $59.0 \pm 10.2$ & $<0.001$ \\
\hline Body mass index, kg/m² & $24.5 \pm 3.1$ & $24.3 \pm 3.0$ & $24.7 \pm 3.2$ & 0.033 \\
\hline $\mathrm{SBP}, \mathrm{mmHg}$ & $118.2 \pm 17 \cdot 3$ & $118.9 \pm 16.2$ & $117.7 \pm 18.0$ & 0.208 \\
\hline $\mathrm{DBP}, \mathrm{mmHg}$ & $71.4 \pm 10.4$ & $74.1 \pm 10.0$ & $69.6 \pm 10.3$ & $<0.001$ \\
\hline Sleep duration, min & $430.9 \pm 76.2$ & $431.4 \pm 76.2$ & $430.6 \pm 76.3$ & 0.861 \\
\hline$<6$ hours/day & $130(9.6)$ & $47(8.6)$ & $83(10.2)$ & \\
\hline 6 to $<7$ hours/day & $301(22.1)$ & $127(23 \cdot 3)$ & $174(21.3)$ & \\
\hline 7 to $<8$ hours/day & $458(33.7)$ & $185(34.0)$ & $273(33.5)$ & \\
\hline 8 to $<9$ hours/day & $319(23.5)$ & $119(21.9)$ & $200(24.5)$ & \\
\hline$\geq 9$ hours/day & $152(11.2)$ & $66(12.1)$ & $86(10.5)$ & \\
\hline \multicolumn{5}{|l|}{ Sleep quality } \\
\hline Sleep difficulty (at least 1/week) & $413(30.4)$ & $108(19.9)$ & $305(37.4)$ & $<0.001$ \\
\hline Sleep awake (at least 1/week) & $506(37.2)$ & $159(29.2)$ & $347(42.5)$ & $<0.001$ \\
\hline Snoring & $672(49.4)$ & $282(51.8)$ & $390(47.8)$ & 0.160 \\
\hline Sleep apnea & $117(8.6)$ & $76(14.0)$ & $41(5 \cdot 0)$ & $<0.001$ \\
\hline Depression & $176(12.9)$ & $56(10.3)$ & $120(14.7)$ & 0.022 \\
\hline Diabetes mellitus & $165(12.1)$ & $84(15 \cdot 4)$ & $81(9.9)$ & 0.003 \\
\hline Hypertension & $516(37 \cdot 9)$ & $212(39.0)$ & $304(37 \cdot 3)$ & 0.561 \\
\hline Hypercholesterolemia & $327(24 \cdot 0)$ & $94(17 \cdot 3)$ & $233(28.6)$ & $<0.001$ \\
\hline History of cardiovascular disease & $103(7.6)$ & $59(10.8)$ & $44(5 \cdot 4)$ & $<0.001$ \\
\hline History of cancer & $53(3.9)$ & $14(2.6)$ & $39(4.8)$ & 0.055 \\
\hline Current drinker & $519(38.2)$ & $324(59 \cdot 6)$ & $195(23.9)$ & $<0.001$ \\
\hline Current smoker & $154(11.3)$ & $136(25.0)$ & $18(2.2)$ & $<0.001$ \\
\hline Regular exercise & $503(37.0)$ & $214(39 \cdot 3)$ & $289(35 \cdot 4)$ & 0.159 \\
\hline Low household income (> 1 million won) & $580(42.6)$ & $197(36.2)$ & $383(46.9)$ & $<0.001$ \\
\hline Single, divorced, or separated & $200(14.7)$ & $29(5 \cdot 3)$ & $171(21.0)$ & $<0.001$ \\
\hline Unemployed or housewives & $446(32.8)$ & $119(21.9)$ & $327(40.1)$ & $<0.001$ \\
\hline Fasting glucose, mg/dL & $98.4 \pm 20.8$ & $101.8 \pm 24.4$ & $96.1 \pm 17.6$ & $<0.001$ \\
\hline Total cholesterol, mg/dL & $191.9 \pm 33.9$ & $184.2 \pm 32.2$ & $197.1 \pm 34.1$ & $<0.001$ \\
\hline HDL-C, mg/dL & $46.3 \pm 11.6$ & $43 \cdot 7 \pm 10.8$ & $48.1 \pm 11.8$ & $<0.001$ \\
\hline Triglycerides, mg/dL & $148.2 \pm 89.5$ & $156.1 \pm 101.2$ & $143.0 \pm 80.4$ & 0.008 \\
\hline hs-CRP, mg/L & $1.7 \pm 5.3$ & $1.9 \pm 7.0$ & $1.5 \pm 3.7$ & 0.196 \\
\hline Blood urea nitrogen, mg/dL & $16.2 \pm 4.8$ & $17.0 \pm 4.9$ & $15 \cdot 7 \pm 4.6$ & $<0.001$ \\
\hline Creatinine, $\mathrm{mg} / \mathrm{dL}$ & $0.9 \pm 0.2$ & $1.1 \pm 0.2$ & $0.9 \pm 0.1$ & $<0.001$ \\
\hline eGFR, $\mathrm{mL} / \mathrm{min} / 1.73 \mathrm{~m}^{2}$ & $74.6 \pm 11.5$ & $76.0 \pm 12.4$ & $73.7 \pm 10.7$ & $<0.001$ \\
\hline Proteinuria $(\geq 1+)$ & $15(1.1)$ & $8(1.5)$ & $7(0.9)$ & 0.430 \\
\hline High creatinine (>1.3 men, > 1.0 women) & $100(7 \cdot 4)$ & $40(7 \cdot 4)$ & $60(7 \cdot 4)$ & 0.084 \\
\hline Low eGFR $\left(<60 \mathrm{~mL} / \mathrm{min} / 1.73 \mathrm{~m}^{2}\right)$ & $115(8.5)$ & $46(8.5)$ & $69(8.5)$ & 0.079 \\
\hline CKD & $125(9.2)$ & $52(9.6)$ & $73(8.9)$ & 0.774 \\
\hline
\end{tabular}

Values are presented as mean \pm SD or number (\%).

SBP, systolic blood pressure; DBP, diastolic blood pressure; HDL-C, high density lipoprotein cholesterol; hs-CRP, high-sensitivity C-reactive protein; eGFR, estimated glomerular filtration rate; CKD, chronic kidney disease. 
men. The prevalence of low eGFR was 8.5\% among both men and women. The overall prevalence of CKD was 9.6\% among men and 8.9\% among women; the difference was not statistically significant. The prevalences of CKD according to stage are presented in Supplementary Table 1.

The general characteristics of the male and female participants are presented according to sleep duration category in Tables 2 and 3. In both men and women, mean age was highest among very long sleepers. In men, individuals with a very long sleep duration were more likely to be short, weigh less, and comprise a higher frequency of unemployed men. Among men, we noted no significant associations between sleep duration and serum creatinine concentration, eGFR, and CKD prevalence. In women, individuals with a very long duration were more likely to have a lower BMI, a higher frequency of menopause, and the lowest household income. Moreover, among women, we observed significantly different distributions for serum creatinine levels, eGFR, and CKD prevalence according to sleep duration. Nevertheless, in both men and women, very long sleepers showed the highest prevalences of CKD.

Table 4 showed the result of the multiple linear regression analysis of the associations between sleep duration and CKD. Adjusted ORs for kidney functions and its components were estimated in separate models with average sleep duration assessed as a continuous variable in one and a categorical variable in the other. Among women, the longest sleep duration was significantly associated with high serum creatinine concentrations, low eGFR, and presence of CKD in the unadjusted model. After additional adjustment for sociodemographic status, socioeconomic status, health behaviors, comorbidities, and sleep quality, the associations remained significant. Women who slept $\geq 9$ hours/day showed significantly increased odds for having high serum creatinine concentrations (OR, 2.936; 95\% CI, 1.176 to 7.326), low eGFR (OR, 3.320; 95\% CI, 1.372 to 8.034), and CKD (OR, 3.112; 95\% CI, 1.315 to 7.363 ), compared to the reference group (sleep duration of 7 to $<8$ hours/day). Among women, for every 1 hour increase in sleep duration per day, there was a $24.6 \%$ increase in the presence of CKD after adjusting for covariates. As a further visual cue, cubic splines were used to display the relationship between average sleep duration and kidney function (Supplementary Fig. 1).
Women who slept more than 8 hours per day exhibited higher serum creatinine concentration and lower eGFR than the reference group. As shown in Fig. 1, the ORs ( $95 \% \mathrm{CI}$ ) for high serum creatinine concentration, low eGFR, and presence of CKD was the highest among female very long sleepers. Among men, there were no significant associations between sleep duration and high serum creatinine concentration, low eGFR, and presence of CKD.

In the sensitivity analysis (Supplementary Tables 2 and 3), we found that sleeping more than 8 hours was significantly associated with CKD (OR, 2.699; 95\% CI, 1.065 to 6.841 ) in women of older age (6o years or older) when stratified according to the median age. Among younger women (less than 60 years), there were no significant associations between sleep duration and kidney function; however, increases in average sleep duration tended to increase the risk for CKD. Additionally, we repeated the logistic regression analysis after excluding participants with hypertension, diabetes mellitus, and a history of cardiovascular disease (Supplementary Table 4), and the association trend was quite consistent.

\section{DISCUSSION}

We investigated the associations between sleep duration and CKD in a Korean adult population. Herein, sleeping more than 9 hours was significantly associated with CKD in women; however, sleep duration was not significantly associated with CKD in men.

In recent decades, the average sleep duration in most individuals has reduced, and many studies have investigated shorter sleep durations and their effects on health. In particular, the average sleep duration of Koreans was 7 hours and 49 minutes per day, which was the lowest among 18 OECD (Organisation for Economic Co-operation and Development) member countries [19]. Notwithstanding, epidemiological evidence of associations between sleep duration and CKD is insufficient. Previous studies reported that sleeping less than 5 hours was associated with proteinuria in the Japanese population $[8,20]$ and higher risk of CKD among the shift workers [13]. More recently, an increasing number of studies have linked prolonged sleep duration with poor health effects $[21,22]$. However, studies have yet to iden- 
Table 2. Characteristics of the study participants according to sleep duration in men $(\mathbf{n}=544)$

\begin{tabular}{|c|c|c|c|c|c|c|c|}
\hline \multirow{2}{*}{ Variable } & \multicolumn{5}{|c|}{ Sleep duration } & \multirow{2}{*}{$p$ value } & \multirow{2}{*}{$\begin{array}{l}p \text { for } \\
\text { trend }\end{array}$} \\
\hline & $<6(\mathrm{n}=47)$ & 6 to $<7(n=127)$ & 7 to $<8(n=185)$ & 8 to $<9(n=119)$ & $\geq 9(n=66)$ & & \\
\hline Age, yr & $59.4 \pm 10.6$ & $59.5 \pm 10.4$ & $61.4 \pm 9.8$ & $62.3 \pm 9.2$ & $65.6 \pm 11.2$ & 0.001 & $<0.001$ \\
\hline Body mass index, kg/m² & $24.2 \pm 3 \cdot 5$ & $24 \cdot 3 \pm 3.0$ & $24.6 \pm 2.9$ & $24.1 \pm 3.1$ & $23.9 \pm 2.7$ & 0.424 & 0.510 \\
\hline $\mathrm{SBP}, \mathrm{mmHg}$ & $117.4 \pm 16.0$ & $118.4 \pm 14.9$ & $119.1 \pm 15.5$ & $119.0 \pm 17 \cdot 3$ & $120.0 \pm 18.4$ & 0.935 & 0.387 \\
\hline DBP, mmHg & $73.2 \pm 10.6$ & $74.1 \pm 10.6$ & $73.7 \pm 9.1$ & $75.1 \pm 9.7$ & $73.6 \pm 11.1$ & 0.765 & 0.714 \\
\hline Sleep duration, min & $290.9 \pm 38.4$ & $368.0 \pm 13.6$ & $428.1 \pm 13 \cdot 3$ & $487.2 \pm 13.2$ & $561.8 \pm 33.1$ & $<0.001$ & $<0.001$ \\
\hline Sleep difficulty (at least 1/week) & $17(36.2)$ & $29(22.8)$ & $31(16.8)$ & $13(10.9)$ & $18(27 \cdot 3)$ & 0.001 & 0.058 \\
\hline Sleep awakeness (at least 1/week) & $17(36.2)$ & $35(27.6)$ & $55(29.7)$ & $33(27 \cdot 7)$ & $19(28.8)$ & 0.840 & 0.573 \\
\hline Snoring & $29(61.7)$ & $68(53 \cdot 5)$ & $91(49.2)$ & $63(52.9)$ & $31(47 \cdot 0)$ & 0.528 & 0.209 \\
\hline Sleep apnea & $8(17.0)$ & $20(15 \cdot 7)$ & $25(13 \cdot 5)$ & $16(13 \cdot 4)$ & $7(10.6)$ & 0.848 & 0.265 \\
\hline Depression & $6(12.8)$ & $8(6.3)$ & $10(5 \cdot 4)$ & $8(6.7)$ & $6(9.1)$ & $0.45^{1}$ & 0.755 \\
\hline Diabetes mellitus & $6(12.8)$ & $14(11.0)$ & $27(14.6)$ & $26(21.8)$ & $11(16.7)$ & 0.194 & 0.069 \\
\hline Hypertension & $21(44 \cdot 7)$ & $42(33.1)$ & $69(37 \cdot 3)$ & $52(43.7)$ & $28(42.4)$ & 0.384 & 0.339 \\
\hline Hypercholesterolemia & $7(14.9)$ & $20(15 \cdot 7)$ & $36(19 \cdot 5)$ & $22(18.5)$ & $9(13 \cdot 6)$ & 0.783 & 0.935 \\
\hline History of cardiovascular disease & $4(8.5)$ & $7(5 \cdot 5)$ & $25(13 \cdot 5)$ & $16(13.4)$ & $7(10.6)$ & 0.185 & 0.152 \\
\hline History of cancer & $2(4 \cdot 3)$ & $1(0.8)$ & $5(2.7)$ & $5(4 \cdot 2)$ & $1(1.5)$ & 0.447 & 0.769 \\
\hline Current drinker & $26(55 \cdot 3)$ & $78(61.4)$ & $117(63.2)$ & $65(54.6)$ & $38(57.6)$ & 0.577 & 0.596 \\
\hline Current smoker & $11(23 \cdot 4)$ & $35(27.6)$ & $44(23.8)$ & $27(22.7)$ & $19(28.8)$ & 0.827 & 0.965 \\
\hline Regular exercise & $18(38.3)$ & $58(45 \cdot 7)$ & $68(36.8)$ & $53(44 \cdot 5)$ & $17(25.8)$ & 0.058 & 0.145 \\
\hline $\begin{array}{l}\text { Low household income } \\
\text { (> } 1 \text { million won) }\end{array}$ & $20(42.6)$ & $48(37.8)$ & $59(31.9)$ & $40(33.6)$ & $30(45 \cdot 5)$ & 0.264 & 0.929 \\
\hline Single, divorced, or separated & $4(8.5)$ & $10(7 \cdot 9)$ & $6(3.2)$ & $3(2.5)$ & $6(9.1)$ & 0.096 & 0.438 \\
\hline Unemployed or housewives & $8(17.0)$ & $23(18.1)$ & $38(20.5)$ & $26(21.8)$ & $24(36.4)$ & 0.040 & 0.009 \\
\hline Fasting glucose, mg/dL & $98.4 \pm 28.7$ & $100.4 \pm 23.8$ & $102.5 \pm 23.5$ & $101.7 \pm 22.4$ & $105.5 \pm 28.5$ & $0.55^{8}$ & 0.115 \\
\hline Total cholesterol, mg/dL & $183.9 \pm 32.0$ & $185.6 \pm 31.2$ & $184.7 \pm 32.0$ & $182.9 \pm 34.0$ & $182.3 \pm 32.3$ & 0.947 & 0.697 \\
\hline HDL-C, mg/dL & $44.0 \pm 10.6$ & $43.7 \pm 11.2$ & $43.9 \pm 10.7$ & $43.9 \pm 11.0$ & $42.5 \pm 10.5$ & 0.911 & 0.490 \\
\hline Triglycerides, mg/dL & $141.4 \pm 80.4$ & $163.1 \pm 114 \cdot 7$ & $158.3 \pm 99.6$ & $156.4 \pm 100.2$ & $147.1 \pm 94.2$ & 0.700 & 0.859 \\
\hline hs-CRP, mg/L & $1.6 \pm 2.3$ & $2.0 \pm 7 \cdot 7$ & $1.5 \pm 2.4$ & $2.8 \pm 12.3$ & $1.3 \pm 1.8$ & 0.516 & 0.967 \\
\hline Blood urea nitrogen, mg/dL & $17 \cdot 4 \pm 4.9$ & $16.5 \pm 4.6$ & $16.9 \pm 4.6$ & $17 \cdot 4 \pm 5 \cdot 6$ & $17 \cdot 6 \pm 5 \cdot 1$ & 0.441 & 0.545 \\
\hline Creatinine, mg/dL & $1.1 \pm 0.1$ & $1.1 \pm 0.2$ & $1.1 \pm 0.2$ & $1.1 \pm 0.2$ & $1.1 \pm 0.2$ & 0.919 & 0.415 \\
\hline eGFR, $\mathrm{mL} / \mathrm{min} / 1.73 \mathrm{~m}^{2}$ & $77.2 \pm 12.4$ & $76.8 \pm 10.7$ & $75.6 \pm 11.5$ & $76.3 \pm 15.1$ & $74.1 \pm 12.6$ & 0.598 & 0.172 \\
\hline Proteinuria $(\geq 1+)$ & o & $2(1.6)$ & $3(1.6)$ & $2(1.7)$ & $1(1.5)$ & 0.941 & 0.624 \\
\hline High creatinine $(>1.3 \mathrm{mg} / \mathrm{dL})$ & $3(6.4)$ & $5(3 \cdot 9)$ & $17(9.2)$ & $10(8.4)$ & $5(7 \cdot 6)$ & 0.501 & 0.324 \\
\hline Low eGFR $\left(<60 \mathrm{~mL} / \mathrm{min} / 1.73 \mathrm{~m}^{2}\right)$ & $3(6.4)$ & $7(5 \cdot 5)$ & $18(9.7)$ & $11(9.2)$ & $7(10.6)$ & 0.634 & 0.197 \\
\hline CKD & $3(6.4)$ & $9(7.1)$ & $20(10.8)$ & $12(10.1)$ & $8(12.1)$ & 0.677 & 0.192 \\
\hline
\end{tabular}

Values are presented as mean \pm SD or number $(\%)$.

SBP, systolic blood pressure; DBP, diastolic blood pressure; HDL-C, high density lipoprotein cholesterol; hs-CRP, high-sensitivity C-reactive protein; eGFR, estimated glomerular filtration rate; CKD, chronic kidney disease.

tify whether these same relationships exists in people who sleep longer.

In the present study of 1360 Koreans, the overall prev- alence of CKD was $9.2 \%$ (9.6\% for men and $8.9 \%$ in women), similar to other results. According to data from the National Health and Nutrition Examination Surveys 
Table 3. Characteristics of the study participants according to sleep duration in women $(n=816)$

\begin{tabular}{|c|c|c|c|c|c|c|c|}
\hline \multirow[b]{2}{*}{ Variable } & \multicolumn{5}{|c|}{ Sleep duration } & \multirow[b]{2}{*}{$p$ value } & \multirow{2}{*}{$\begin{array}{l}p \text { for } \\
\text { trend }\end{array}$} \\
\hline & $<6(\mathrm{n}=83)$ & $\begin{array}{l}6 \text { to }<7 \\
(n=174)\end{array}$ & $\begin{array}{l}7 \text { to }<8 \\
(\mathrm{n}=273)\end{array}$ & $\begin{array}{l}8 \text { to }<9 \\
(n=200)\end{array}$ & $\geq 9(\mathrm{n}=86)$ & & \\
\hline Age, yr & $59.8 \pm 10.0$ & $57.7 \pm 10.6$ & $57.2 \pm 9.8$ & $60.1 \pm 9.6$ & $63.6 \pm 10.5$ & $<0.001$ & 0.004 \\
\hline Body mass index, kg/m² & $25.1 \pm 3.2$ & $25 \cdot 0 \pm 3 \cdot 3$ & $24.4 \pm 3.2$ & $24.8 \pm 3.1$ & $24.1 \pm 3.2$ & 0.085 & 0.030 \\
\hline $\mathrm{SBP}, \mathrm{mmHg}$ & $117.6 \pm 19.9$ & $117.4 \pm 17.7$ & $116.2 \pm 17.6$ & $119 \cdot 4 \pm 17 \cdot 2$ & $119 \cdot 5 \pm 19 \cdot 7$ & 0.339 & 0.385 \\
\hline $\mathrm{DBP}, \mathrm{mmHg}$ & $69.4 \pm 11.4$ & $69.3 \pm 10.3$ & $69.2 \pm 10.3$ & $70.2 \pm 10.0$ & $70.3 \pm 10.1$ & 0.795 & 0.501 \\
\hline Sleep duration, $\min$ & $297 \cdot 3 \pm 40.9$ & $368.4 \pm 13.6$ & $428.0 \pm 13 \cdot 5$ & $484.8 \pm 11.1$ & $567.6 \pm 42.0$ & $<0.001$ & $<0.001$ \\
\hline Sleep difficulty (at least 1/week) & $45(54.2)$ & $70(40.2)$ & $92(33.7)$ & $66(33.0)$ & $32(37.2)$ & 0.008 & 0.007 \\
\hline Sleep awakeness (at least 1/week) & $49(59.0)$ & $76(43.7)$ & $103(37 \cdot 7)$ & $74(37.0)$ & $45(52.3)$ & 0.001 & 0.140 \\
\hline Snoring & $43(51.8)$ & $93(53 \cdot 4)$ & $124(45 \cdot 4)$ & $91(45 \cdot 5)$ & $39(45 \cdot 3)$ & 0.405 & 0.118 \\
\hline Sleep apnea & $2(2.4)$ & $10(5 \cdot 7)$ & $11(4 \cdot 0)$ & $13(6.5)$ & $5(5.8)$ & 0.563 & 0.296 \\
\hline Depression & $14(16.9)$ & $22(12.6)$ & $26(9.5)$ & $28(14.0)$ & $12(14.0)$ & 0.375 & 0.852 \\
\hline Diabetes mellitus & $9(10.8)$ & $20(11.5)$ & $25(9.2)$ & $16(8.0)$ & $11(12.8)$ & 0.671 & 0.743 \\
\hline Hypertension & $39(47.0)$ & $73(42.0)$ & $84(30.8)$ & $70(35 \cdot 0)$ & $38(44.2)$ & 0.017 & 0.280 \\
\hline Hypercholesterolemia & $25(30.1)$ & $56(32.2)$ & $72(26.4)$ & $61(30.5)$ & $19(22.1)$ & 0.407 & 0.270 \\
\hline $\begin{array}{l}\text { History of cardiovascular dis- } \\
\text { ease }\end{array}$ & $2(2.4)$ & $8(4.6)$ & $14(5.1)$ & $14(7 \cdot 0)$ & $6(7 \cdot 0)$ & 0.535 & 0.093 \\
\hline History of cancer & $2(2.4)$ & $9(5.2)$ & $15(5 \cdot 5)$ & $9(4 \cdot 5)$ & $4(4 \cdot 7)$ & 0.839 & 0.721 \\
\hline Menopause & $67(80.7)$ & $127(73.0)$ & $201(73.6)$ & $168(84.0)$ & $76(88.4)$ & 0.004 & 0.011 \\
\hline Current drinker & $20(24.1)$ & $39(22.4)$ & $72(26.4)$ & $45(22.5)$ & $19(22.1)$ & 0.826 & 0.792 \\
\hline Current smoker & 0 & $2(1.1)$ & $11(4 \cdot 0)$ & $5(2.5)$ & 0 & 0.061 & 0.630 \\
\hline Regular exercise & $32(38.6)$ & $70(40.2)$ & $97(35 \cdot 5)$ & $64(32.0)$ & $26(30.2)$ & 0.386 & 0.058 \\
\hline $\begin{array}{l}\text { Low household income (> } 1 \text { mil- } \\
\text { lion won) }\end{array}$ & $41(49 \cdot 4)$ & $73(42.0)$ & $114(41.8)$ & $103(51.5)$ & $52(60.5)$ & 0.012 & 0.022 \\
\hline Single, divorced, or separated & $20(24.1)$ & $33(19.0)$ & $47(17.2)$ & $43(21.5)$ & $28(32.6)$ & 0.037 & 0.143 \\
\hline Unemployed or housewives & $33(39.8)$ & $75(43.1)$ & $108(39.6)$ & $71(35 \cdot 5)$ & $40(46.5)$ & 0.416 & 0.859 \\
\hline Fasting glucose, mg/dL & $100.1 \pm 27.0$ & $96.2 \pm 20.0$ & $95.6 \pm 15.7$ & $94.3 \pm 12.2$ & $97 \cdot 3 \pm 16.4$ & 0.134 & 0.217 \\
\hline Total cholesterol, mg/dL & $205.0 \pm 31.2$ & $196.4 \pm 37.1$ & $193.7 \pm 30.6$ & $200.8 \pm 35.2$ & $193 \cdot 5 \pm 37.0$ & 0.033 & 0.064 \\
\hline HDL-C, mg/dL & $49.7 \pm 13.2$ & $48.3 \pm 10.7$ & $48.3 \pm 11.8$ & $47 \cdot 4 \pm 11.3$ & $47.2 \pm 13 \cdot 5$ & 0.571 & 0.131 \\
\hline Triglycerides, mg/dL & $137 \cdot 0 \pm 61.1$ & $139 \cdot 9 \pm 92.4$ & $142.5 \pm 81.6$ & $147 \cdot 6 \pm 77 \cdot 6$ & $145 \cdot 4 \pm 74.8$ & 0.835 & 0.390 \\
\hline hs-CRP, mg/L & $1.4 \pm 2.7$ & $1.4 \pm 2.7$ & $1.4 \pm 2.9$ & $1.5 \pm 2.7$ & $2.3 \pm 8.0$ & 0.332 & 0.101 \\
\hline Blood urea nitrogen, mg/dL & $15 \cdot 7 \pm 4 \cdot 5$ & $16.0 \pm 4.9$ & $15 \cdot 3 \pm 4 \cdot 1$ & $15 \cdot 6 \pm 4.6$ & $16.4 \pm 5.4$ & 0.300 & 0.416 \\
\hline Creatinine, mg/dL & $0.9 \pm 0.1$ & $0.9 \pm 0.1$ & $0.8 \pm 0.1$ & $0.9 \pm 0.1$ & $0.9 \pm 0.2$ & 0.006 & 0.023 \\
\hline $\mathrm{eGFR}, \mathrm{mL} / \mathrm{min} / 1.73 \mathrm{~m}^{2}$ & $73.6 \pm 10.1$ & $73.8 \pm 11.3$ & $75 \cdot 3 \pm 10.2$ & $72.7 \pm 9.2$ & $70.5 \pm 13.8$ & 0.003 & 0.039 \\
\hline Proteinuria $(\geq 1+)$ & o & $1(0.6)$ & $2(0.7)$ & $3(1.5)$ & $1(1.2)$ & 0.732 & 0.208 \\
\hline High creatinine (> $1.0 \mathrm{mg} / \mathrm{dL})$ & $2(2.4)$ & $15(8.6)$ & $12(4.4)$ & $17(8.5)$ & $14(16.3)$ & 0.002 & 0.005 \\
\hline Low eGFR $\left(<60 \mathrm{~mL} / \mathrm{min} / 1.73 \mathrm{~m}^{2}\right)$ & $5(6.0)$ & $15(8.6)$ & $13(4.8)$ & $19(9 \cdot 5)$ & $17(19.8)$ & 0.001 & 0.005 \\
\hline CKD & $5(6.0)$ & $15(8.6)$ & $14(5.1)$ & $22(11.0)$ & $17(19.8)$ & 0.001 & 0.002 \\
\hline
\end{tabular}

Values are presented as mean \pm SD or number (\%).

SBP, systolic blood pressure; DBP, diastolic blood pressure; HDL-C, high density lipoprotein cholesterol; hs-CRP, high-sensitivity C-reactive protein; eGFR, estimated glomerular filtration rate; CKD, chronic kidney disease. 
Table 4. Association between sleep duration and chronic kidney disease criteria

\begin{tabular}{|c|c|c|c|c|}
\hline Sleep duration & Total no. & No. $(\%)$ & Unadjusted OR (95\% CI) & Adjusted OR $(95 \% \mathrm{CI})^{\mathrm{a}}$ \\
\hline \multicolumn{5}{|l|}{ High creatinine } \\
\hline \multicolumn{5}{|l|}{ Men } \\
\hline Continuous, hr/day ${ }^{b}$ & 544 & $52(9.6)$ & $1.114(0.864-1.436)$ & $1.099(0.832-1.452)$ \\
\hline \multicolumn{5}{|l|}{ Categorical, hr/day } \\
\hline$<6$ & 47 & $3(6.4)$ & $0.674(0.189-2.403)$ & $0.608(0.146-2.534)$ \\
\hline 6 to $<7$ & 127 & $5(3.9)$ & $0.405\left(0.145^{-1.128}\right)$ & $0.265(0.079-0.893)$ \\
\hline 7 to $<8$ & 185 & $17(9.2)$ & 1.000 & 1.000 \\
\hline 8 to $<9$ & 119 & $10(8.4)$ & $0.907(0.400-2.053)$ & $1.011(0.404-2.534)$ \\
\hline$\geq 9$ & 66 & $5(7.6)$ & $0.810(0.287-2.290)$ & $0.541(0.164-1.780)$ \\
\hline \multicolumn{5}{|l|}{ Women } \\
\hline Continuous, hr/day ${ }^{\mathrm{b}}$ & 816 & $73(8.9)$ & $1.324(1.078-1.625)$ & $1.258(1.012-1.564)$ \\
\hline \multicolumn{5}{|l|}{ Categorical, hr/day } \\
\hline$<6$ & 83 & $2(2.4)$ & $0.537(0.118-2.449)$ & $0.476(0.097-2.341)$ \\
\hline 6 to $<7$ & 174 & $15(8.6)$ & $2.052(0.937-4.495)$ & $1.767(0.743-4.202)$ \\
\hline 7 to $<8$ & 273 & $12(4 \cdot 4)$ & 1.000 & 1.000 \\
\hline 8 to $<9$ & 200 & $17(8.5)$ & $2.020(0.942-4.332)$ & $1.703(0.730-3.972)$ \\
\hline$\geq 9$ & 86 & $14(16.3)$ & $4.229(1.874-9.545)$ & $2.936(1.176-7.326)$ \\
\hline \multicolumn{5}{|l|}{ Low eGFR } \\
\hline \multicolumn{5}{|l|}{ Men } \\
\hline Continuous, hr/day ${ }^{b}$ & 544 & $52(9.6)$ & $1.190(0.938-1.511)$ & $1.145(0.878-1.494)$ \\
\hline \multicolumn{5}{|l|}{ Categorical, hr/day } \\
\hline$<6$ & 47 & $3(6.4)$ & $0.633(0.178-2.245)$ & $0.514(0.115-2.296)$ \\
\hline 6 to $<7$ & 127 & $7(5 \cdot 5)$ & $0.541(0.219-1.336)$ & $0.493(0.177-1.369)$ \\
\hline 7 to $<8$ & 185 & $18(9.7)$ & 1.000 & 1.000 \\
\hline 8 to $<9$ & 119 & $11(9.2)$ & $0.945(0.430-2.078)$ & $1.131(0.460-2.782)$ \\
\hline$\geq 9$ & 66 & $7(10.6)$ & $1.101(0.438-2.768)$ & $0.709(0.236-2.132)$ \\
\hline \multicolumn{5}{|l|}{ Women } \\
\hline Continuous, hr/day ${ }^{\mathrm{b}}$ & 816 & $73(8.9)$ & $1.301(1.073-1.579)$ & $1.222(0.994-1.503)$ \\
\hline \multicolumn{5}{|l|}{ Categorical, hr/day } \\
\hline$<6$ & 83 & $5(6.0)$ & $1.282(0.443-3.708)$ & $1.161(0.362-3.719)$ \\
\hline 6 to $<7$ & 174 & $15(8.6)$ & $1.887(0.875-4.069)$ & $1.641(0.688-3.916)$ \\
\hline 7 to $<8$ & 273 & $13(4.8)$ & 1.000 & 1.000 \\
\hline 8 to $<9$ & 200 & $19(9 \cdot 5)$ & $2.099(1.011-4.359)$ & $1.692(0.743-3.849)$ \\
\hline$\geq 9$ & 86 & $17(19.8)$ & $4.928(2.283-10.636)$ & $3.320(1.372-8.034)$ \\
\hline \multicolumn{5}{|l|}{ Chronic kidney disease } \\
\hline \multicolumn{5}{|l|}{ Men } \\
\hline Continuous, hr/day ${ }^{\mathrm{b}}$ & 544 & $52(9.6)$ & $1.182(0.943-1.482)$ & $1.091(0.845-1.410)$ \\
\hline \multicolumn{5}{|l|}{ Categorical, hr/day } \\
\hline$<6$ & 47 & $3(6.4)$ & $0.563(0.160-1.980)$ & $0.453(0.102-2.009)$ \\
\hline 6 to $<7$ & 127 & $9(7.1)$ & $0.629(0.277-1.431)$ & $0.603\left(0.235^{-1.545)}\right.$ \\
\hline 7 to $<8$ & 185 & $20(10.8)$ & 1.000 & 1.000 \\
\hline 8 to $<9$ & 119 & $12(10.1)$ & $0.925(0.434-1.970)$ & $1.041(0.435-2.489)$ \\
\hline
\end{tabular}


Table 4. continued

\begin{tabular}{lcccc}
\hline Sleep duration & Total no. & No. $(\%)$ & Unadjusted OR (95\% CI) & Adjusted OR (95\% CI) $^{\mathrm{a}}$ \\
\hline $\begin{array}{l}\text { Women } \\
\text { Continuous, hr/day }\end{array}$ & 66 & $8(12.1)$ & $1.138(0.475-2.724)$ & $0.591(0.199-1.760)$ \\
\hline $\begin{array}{l}\text { Categorical, hr/day } \\
<6\end{array}$ & 816 & $73(8.9)$ & $1.319(1.092-1.592)$ & $1.246(1.019-1.523)$ \\
\hline to $<7$ & 83 & $5(6.0)$ & $1.186(0.414-3.396)$ & $1.076(0.341-3.393)$ \\
7 to $<8$ & 174 & $15(8.6)$ & $1.745(0.821-3.712)$ & $1.475(0.635-3.428)$ \\
8 to $<9$ & 273 & $14(5.1)$ & 1.000 & 1.000 \\
$\geq 9$ & 200 & $22(11.0)$ & $2.287(1.139-4.589)$ & $1.929(0.885-4.204)$ \\
\hline
\end{tabular}

OR, odds ratio; CI, confidence interval; eGFR, estimated glomerular filtration rate.

${ }^{a}$ Adjusted model: adjusted for sex, age, body mass index, systolic blood pressure, smoking, alcohol, exercise, diabetes mellitus, hypercholesterolemia, depression, history of cancer, menopause, socioeconomic status (household income, marital status, and working status), and sleep quality (sleep difficulty, sleep awakeness, snoring, and sleep apnea).

${ }^{\mathrm{b}}$ Continuous model: association analysis on total sleep duration as a continuous variable.

(NHANES) in the United States, the overall prevalences of CKD were $10.0 \%$ in 1988 to 1994 and $13.1 \%$ in 1999 to 2004 [4]. The overall prevalences of CKD in Asian adult populations are $12.9 \%$ in Japan [5] and $10.8 \%$ in China [6]. The overall prevalence of CKD in the Korea National Health and Nutrition Examination Survey (KNHANES) I to IV ranges from $3.9 \%$ to $7.9 \%$ in men and $6.3 \%$ to $12.0 \%$ in women, respectively [7].

Although plausible biological mechanisms for the relationship between short sleep duration and CKD have been proposed, it is less clear as to how long sleep duration is associated with CKD prevalence. The temporal associations between sleep duration and CKD are likely to be bi-directional; nevertheless, as long sleep duration could be either an initial symptom or a consequence of unmeasured diseases and conditions among CKD patients, any causality cannot be inferred from the present data. Previous studies have also suggested that the association of long sleep duration with CKD could be explained by residual confounding and comorbidities $[3,23]$. Accordingly, potential confounders could predispose individuals to both long sleep duration and poor kidney function. Thus, to reduce the effects of unmeasured confounders on our results, we adjusted for sociodemographic status, socioeconomic status, health behaviors, comorbidities, and sleep quality, which may link long sleep duration to CKD. Even after adjusting for these confounders, the association between a long sleep duration and CKD remained significant. Notwithstand- ing, the adverse health outcomes of long sleep duration and CKD share a few commonalities, including associations with old age, diabetes, established cardiovascular disease, high blood pressure, obesity, and smoking [24]. Interestingly, sleep duration has been shown to be associated with cardiometabolic diseases by worsening kidney hemodynamics and may lead to the development of glomerulosclerosis and glomerulomegaly [24-30].

A number of mechanisms could potentially mediate the associations between long sleep duration and kidney dysfunction, encompassing both biological and socioeconomic factors. Inflammation has been shown to affect the relationship between long sleep duration and kidney dysfunction [12]. Indeed, several studies have indicated that immune dysfunction may lead to progressive kidney dysfunction through activation of the renin-angiotensin-aldosterone system, which is a wellknown regulator of blood pressure and a progression factor in CKD [27,29,31-33]. In addition, lower socioeconomic status could be detrimental to CKD [23,34]. In the Nurses' Health Study II, women who had never married, lived alone, were unemployed, had low household income, and were of low societal status were more likely to sleep longer [23]. Meanwhile, lower socioeconomic status could increase the risk for CKD due to both a lack of access to health care and receipt of poorer quality of care [23]. For people with CKD, lower socioeconomic status may contribute to poor control of hypertension and diabetes mellitus due to lack of understanding of 

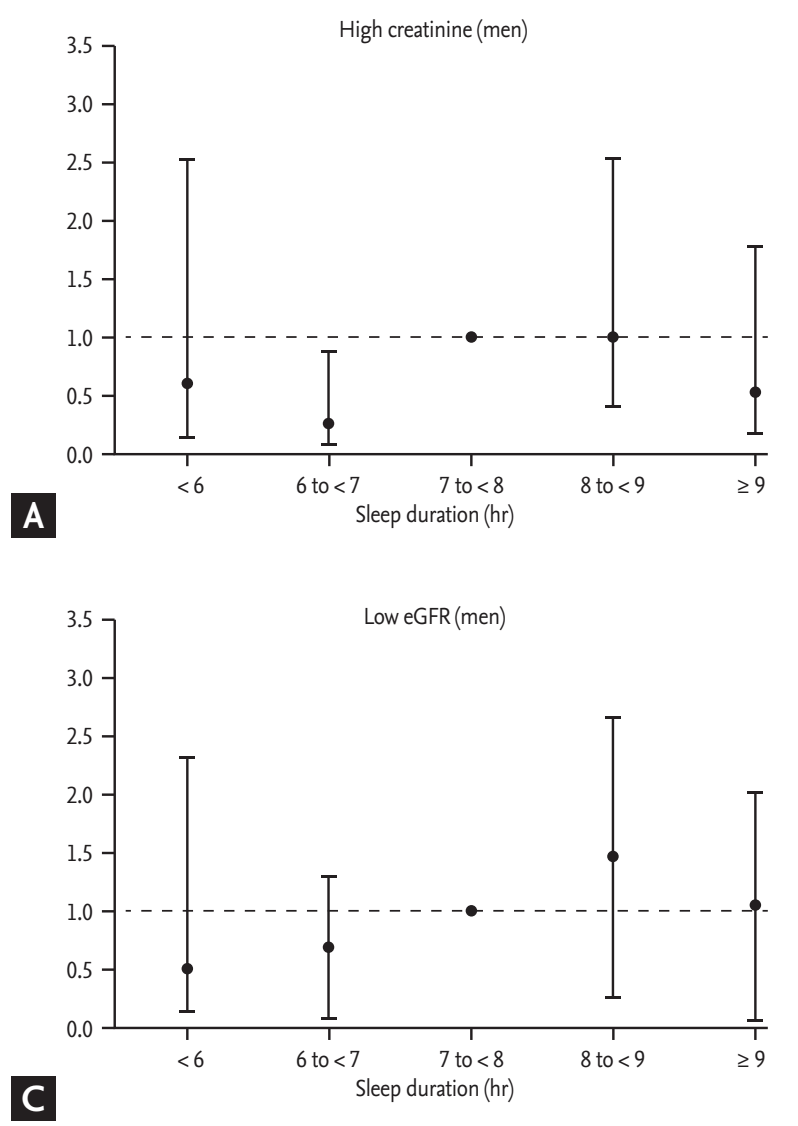

C

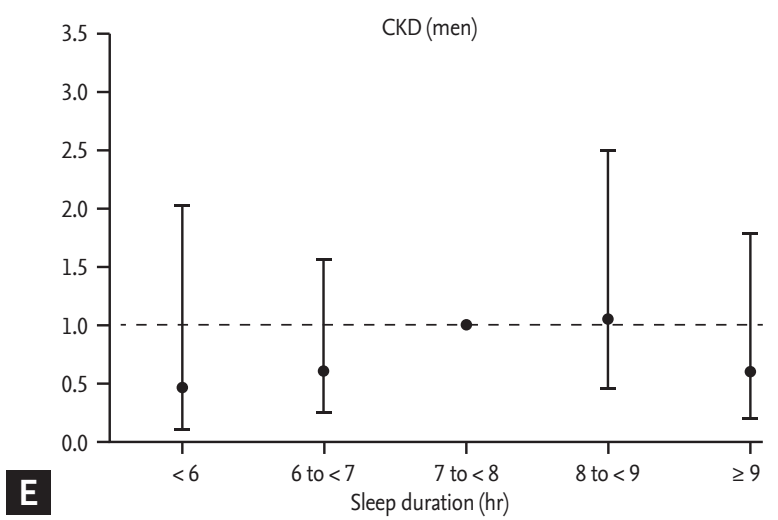

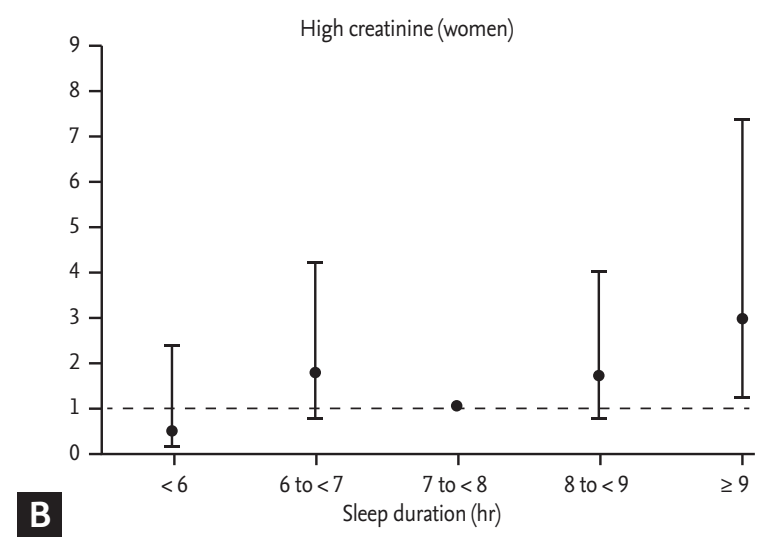
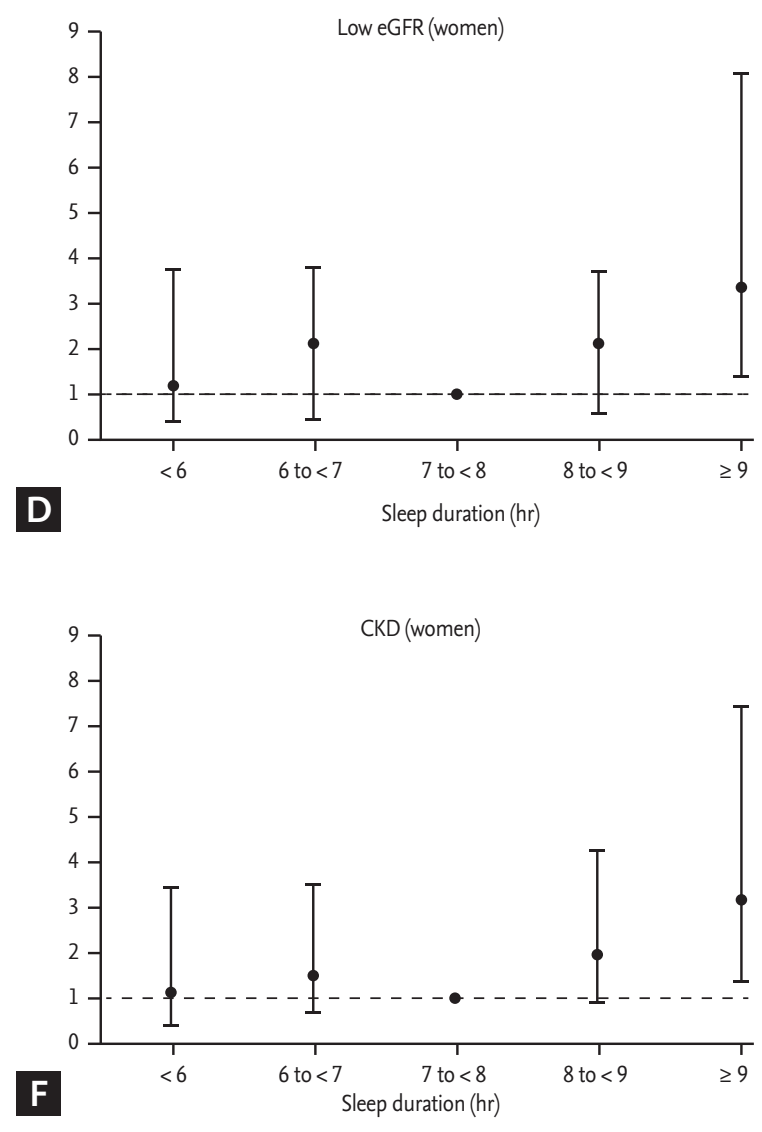

Figure 1. Association between sleep duration and chronic kidney disease (CKD). (A) Association between sleep duration and high creatinine in men. (B) Association between sleep duration and high creatinine in women. (C) Association between sleep duration and low estimated glomerular filtration rate (eGFR) in men. (D) Association between sleep duration and low eGFR in women. (E) Association between sleep duration and CKD in men. (F) Association between sleep duration and CKD in women. CKD was defined as the presence of at least one of the following factors: eGFR than $60 \mathrm{~mL} / \mathrm{min} / 1.73 \mathrm{~m}^{2}$ or proteinuria. Adjusted model: adjusted for sex, age, body mass index, systolic blood pressure, smoking, alcohol, physical activity, diabetes mellitus, hypercholesterolemia, depression, history of cardiovascular disease, history of cancer, menopause, socioeconomic status (household income, marital status, and working status), and sleep quality (sleep difficulty, sleep awakeness, snoring, and sleep apnea).

the disease or inadequate treatment [34]. Finally, longer hours spent in bed lead to reduced physical activity and could induce a prothrombotic state [23]. Increased phys- ical activity exerts a protective effect against CKD by reducing cardiometabolic risk factors [9,28]. In fact, an experimental study found that repeated exercise increased 
GFR along with a reduction in renal mass in mice [35].

There are several limitations to this study that should be noted. As noted above, the main limitation of the present study relates to its causal relationship between the sleep duration and CKD cannot be confirmed due to the cross-sectional design of the study. Although we controlled for several potential confounders in our statistical models, residual confounding effects may have been present. Second, sleep habits were measured by a self-reported questionnaire and were not objectively confirmed. In future studies, objective measurements, including actigraphy and polysomnography to assess quality and quantity of sleep, would be needed. Third, we did not use 24-hour urinary albumin excretion, which is the gold standard test for assessing proteinuria. Instead, we measured proteinuria by a semiquantitative dipstick test, which has lower sensitivity and specificity, compared with urinary albumin test [23]. Finally, we did not exclude individuals who reported a history of chronic diseases, such as hypertension, diabetes mellitus, and cardiovascular disease, which may have influenced the results. Further longitudinal analyses are required to delineate the possible role of long sleep duration in CKD among Korean adults.

In summary, we suggest that very long sleep duration is associated with CKD in community-dwelling Korean women. Longitudinal analyses are required to clarify the roles of sleep quantity, as well as sleep quality, in maintaining and improving overall health among CKD patients. As sleep duration is a potentially modifiable risk factor, our results may help health advisors with preventing and delaying the progression of CKD.

\section{KEY MESSAGE}

1. Very long sleep duration (more than 9 hours) was significantly associated with chronic kidney disease (CKD) in community-dwelling Korean women.

2. Among women, for every one hour increase in sleep duration per day, there was a $24.6 \%$ increase in the presence of CKD.

\section{Conflict of interest}

No potential conflict of interest relevant to this article was reported.

\section{Acknowledgments}

This work was supported by the Research Program funded by the Korea Centers for Disease Control and Prevention (2010-E71003-00, 2011-E71002-00) and the Korean Health Technology R\&D Project, Ministry of Health and Welfare (HI13Co715).

\section{REFERENCES}

1. Cappuccio FP, D’Elia L, Strazzullo P, Miller MA. Sleep duration and all-cause mortality: a systematic review and meta-analysis of prospective studies. Sleep 2010;33:585592.

2. Knutson KL. Sleep duration and cardiometabolic risk: a review of the epidemiologic evidence. Best Pract Res Clin Endocrinol Metab 2010;24:731-743.

3. Cappuccio FP, Cooper D, D'Elia L, Strazzullo P, Miller MA. Sleep duration predicts cardiovascular outcomes: a systematic review and meta-analysis of prospective studies. Eur Heart J 2011;32:1484-1492.

4. Coresh J, Selvin E, Stevens LA, et al. Prevalence of chronic kidney disease in the United States. JAMA 2007;298:20382047 .

5. Imai E, Horio M, Watanabe T, et al. Prevalence of chronic kidney disease in the Japanese general population. Clin Exp Nephrol 2009;13:621-630.

6. Zhang L, Wang F, Wang L, et al. Prevalence of chronic kidney disease in China: a cross-sectional survey. Lancet 2012;379:815-822.

7. Kang HT, Lee J, Linton JA, Park BJ, Lee YJ. Trends in the prevalence of chronic kidney disease in Korean adults: the Korean National Health and Nutrition Examination Survey from 1998 to 2009. Nephrol Dial Transplant 2013;28:927-936.

8. Fujibayashi K, Fukuda H, Yokokawa H, et al. Associations between healthy lifestyle behaviors and proteinuria and the estimated glomerular filtration rate (eGFR). J Atheroscler Thromb 2012;19:932-940.

9. Stengel B, Tarver-Carr ME, Powe NR, Eberhardt MS, Brancati FL. Lifestyle factors, obesity and the risk of chronic kidney disease. Epidemiology 2003;14:479-487.

10. Iliescu EA, Yeates KE, Holland DC. Quality of sleep in patients with chronic kidney disease. Nephrol Dial Transplant 2004;19:95-99.

11. Ozkok A, Kanbay A, Odabas AR, Covic A, Kanbay M. 
Obstructive sleep apnea syndrome and chronic kidney disease: a new cardiorenal risk factor. Clin Exp Hypertens 2014;36:211-216.

12. Ohkuma T, Fujii H, Iwase M, et al. Association between sleep duration and urinary albumin excretion in patients with type 2 diabetes: the Fukuoka diabetes registry. PLoS One 2013;8:e78968.

13. Sasaki S, Yoshioka E, Saijo Y, Kita T, Tamakoshi A, Kishi R. Short sleep duration increases the risk of chronic kidney disease in shift workers. J Occup Environ Med 2014;56:1243-1248.

14. Jo SA, Park MH, Jo I, Ryu SH, Han C. Usefulness of Beck Depression Inventory (BDI) in the Korean elderly population. Int J Geriatr Psychiatry 2007;22:218-223.

15. American Diabetes Association. Diagnosis and classification of diabetes mellitus. Diabetes Care 2011;34 Suppl 1:S62-S69.

16. Son JI, Chin SO, Woo JT; The Committee for Developing Treatment Guidelines for Dyslipidemia, Korean Society of Lipidology and Atherosclerosis (KSLA). Treatment guidelines for dyslipidemia: summary of the expanded second version. J Lipid Atheroscler 2012;1:45-59.

17. Levey AS, Bosch JP, Lewis JB, Greene T, Rogers N, Roth D. A more accurate method to estimate glomerular filtration rate from serum creatinine: a new prediction equation. Modification of Diet in Renal Disease Study Group. Ann Intern Med 1999;130:461-470.

18. National Kidney Foundation. K/DOQI clinical practice guidelines for chronic kidney disease: evaluation, classification, and stratification. Am J Kidney Dis 2002;39(2 Suppl 1):S1-S266.

19. Organisation for Economic Co-operation and Development (OECD). Society at a Glance 2009. Paris: OECD Publishing, 2009.

20. Yamamoto R, Nagasawa Y, Iwatani H, et al. Self-reported sleep duration and prediction of proteinuria: a retrospective cohort study. Am J Kidney Dis 2012;59:343-355.

21. Kripke DF, Garfinkel L, Wingard DL, Klauber MR, Marler MR. Mortality associated with sleep duration and insomnia. Arch Gen Psychiatry 2002;59:131-136.

22. Patel SR, Ayas NT, Malhotra MR, et al. A prospective study of sleep duration and mortality risk in women. Sleep 2004;27:440-444.
23. Patel SR, Malhotra A, Gottlieb DJ, White DP, Hu FB. Correlates of long sleep duration. Sleep 2006;29:881-889.

24. Kidney Health Australia. Chronic Kidney Disease (CKD) Management in General Practice. 2nd ed. Melbourne: Kidney Health Australia, 2012.

25. Chen J, Muntner P, Hamm LL, et al. Insulin resistance and risk of chronic kidney disease in nondiabetic US adults. J Am Soc Nephrol 2003;14:469-477.

26. Wahba IM, Mak RH. Obesity and obesity-initiated metabolic syndrome: mechanistic links to chronic kidney disease. Clin J Am Soc Nephrol 2007;2:550-562.

27. Fogo AB. Mechanisms of progression of chronic kidney disease. Pediatr Nephrol 2007;22:2011-2022.

28. Morgado E, Neves PL. Hypertension and chronic kidney disease: cause and consequence-therapeutic considerations. In: Babaei H, ed. Antihypertensive Drugs. InTech, 2012:45-66.

29. Wilcox CS, Welch WJ, Murad F, et al. Nitric oxide synthase in macula densa regulates glomerular capillary pressure. Proc Natl Acad Sci U S A 1992;89:11993-11997.

30. Dengel DR, Goldberg AP, Mayuga RS, Kairis GM, Weir MR. Insulin resistance, elevated glomerular filtration fraction, and renal injury. Hypertension 1996;28:127-132.

31. Remuzzi G, Perico N, Macia M, Ruggenenti P. The role of renin-angiotensin-aldosterone system in the progression of chronic kidney disease. Kidney Int Suppl 2005;99:S57-S65.

32. Westhuyzen J, Healy H. Review: biology and relevance of $\mathrm{C}$-reactive protein in cardiovascular and renal disease. Ann Clin Lab Sci 2000;30:133-143.

33. Yang T, Chou YC, Chu CH, et al. Metabolic syndrome and $\mathrm{C}$-reactive protein concentration as independent correlates of chronic kidney disease. Endocr Res 2014;39:9498.

34. Plantinga LC, Johansen KL, Schillinger D, Powe NR. Lower socioeconomic status and disability among US adults with chronic kidney disease, 1999-2008. Prev Chronic Dis 2012;9:E12.

35. Averbukh Z, Marcus E, Berman S, et al. Effect of exercise training on glomerular filtration rate of mice with various degrees of renal mass reduction. Am J Nephrol 1992;12:174-178. 
Supplementary Table 1. Prevalence of indicators of CKD in the study population $(n=1,360)$

\begin{tabular}{|c|c|c|c|c|c|c|c|}
\hline \multirow{2}{*}{$\mathrm{eGFR}, \mathrm{mL} / \mathrm{min} / 1.73 \mathrm{~m}^{2}$} & \multirow{2}{*}{ No. $(\%)^{\mathrm{a}}$} & \multicolumn{4}{|c|}{ Proteinuria, $\mathrm{n}(\%)^{\mathrm{b}}$} & \multicolumn{2}{|c|}{$\mathrm{CKD}^{\mathrm{d}}$} \\
\hline & & Unknown $^{\mathrm{c}}$ & Negative $^{c}$ & Trace $^{c}$ & Positive $(\geq 1+)^{c}$ & Stage & No. $(\%)^{c}$ \\
\hline Total & $1,360(100.0)$ & $7(0.5)$ & $1,296(95 \cdot 3)$ & $42(3.1)$ & $15(1.1)$ & All & $125(9.2)$ \\
\hline$\geq 90$ & $105(7 \cdot 7)$ & 0 & $101(96.2)$ & $3(2.9)$ & $1(1.0)$ & 1 & $1(0.1)$ \\
\hline $60-89$ & $1,140(83.8)$ & $3(0.3)$ & $1,098(96.3)$ & $30(2.6)$ & $9(0.8)$ & 2 & $9(0.7)$ \\
\hline $30-59$ & $111(8.2)$ & $2(1.8)$ & $95(85.6)$ & $9(8.1)$ & $5(4 \cdot 5)$ & 3 & $111(8.2)$ \\
\hline $15-29$ & $\mathrm{O}$ & o & $\mathrm{O}$ & o & $\mathrm{O}$ & 4 & O \\
\hline$<15$ & $4(0.3)$ & $2(50.0)$ & $2(50.0)$ & 0 & $\mathrm{O}$ & 5 & $4(0.3)$ \\
\hline Men & $544(100.0)$ & $2(0.4)$ & $504(92.6)$ & $30(5 \cdot 5)$ & $8(1.5)$ & All & $52(9.6)$ \\
\hline$\geq 90$ & $61(11.2)$ & o & $57(93.4)$ & $3(4 \cdot 9)$ & $1(1.6)$ & 1 & $1(0.2)$ \\
\hline $60-89$ & $437(80.3)$ & $1(0.2)$ & $409(93.6)$ & $22(5.0)$ & $5(1.1)$ & 2 & $5(0.9)$ \\
\hline $30-59$ & $44(8.1)$ & o & $37(84.1)$ & $5(11.4)$ & $2(4 \cdot 5)$ & 3 & $44(8.1)$ \\
\hline $15-29$ & o & o & $\mathrm{O}$ & 0 & $\mathrm{O}$ & 4 & o \\
\hline$<15$ & $2(0.4)$ & $1(50.0)$ & $1(50.0)$ & 0 & 0 & 5 & $2(0.4)$ \\
\hline Women & $816(100.0)$ & $5(0.6)$ & $792(97.1)$ & $12(1.5)$ & $7(0.9)$ & All & $73(8.9)$ \\
\hline$\geq 90$ & $44(5.4)$ & 0 & $44(100.0)$ & 0 & o & 1 & 0 \\
\hline $6 o-89$ & $703(86.2)$ & $2(0.3)$ & $689(98.0)$ & $8(1.1)$ & $4(0.6)$ & 2 & $4(0.5)$ \\
\hline $30-59$ & $67(8.2)$ & $2(3.0)$ & $58(86.6)$ & $4(6.0)$ & $3(4 \cdot 5)$ & 3 & $67(8.2)$ \\
\hline $15-29$ & o & o & o & 0 & o & 4 & o \\
\hline$<15$ & $2(0.3)$ & $1(50.0)$ & $1(50.0)$ & 0 & 0 & 5 & $2(0.2)$ \\
\hline
\end{tabular}

CKD, chronic kidney disease; eGFR, estimated glomerular filtration rate.

${ }^{a}$ Number of participants across each kidney function category.

${ }^{\mathrm{b}}$ Defined as proteinuria $1+$ or more based on a dipstick urine test.

${ }^{\mathrm{c}}$ Number of participants with corresponding indicators of CKD across each kidney function category.

${ }^{\mathrm{d}}$ Defined as the presence of at least one of the following factors: eGFR less than $60 \mathrm{~mL} / \mathrm{min} / 1.73 \mathrm{~m}^{2}$ or proteinuria. 
Supplementary Table 2. Age-specific association between sleep duration and CKD criteria in men $(\mathbf{n}=544)$

\begin{tabular}{|c|c|c|c|c|}
\hline \multirow{2}{*}{ Sleep duration } & \multicolumn{2}{|c|}{ Unadjusted OR (95\% CI) } & \multicolumn{2}{|c|}{ Adjusted OR $(95 \% \text { CI })^{\mathrm{a}}$} \\
\hline & Age $<64(n=295)$ & Age $\geq 64(n=249)$ & Age $<64(n=295)$ & Age $\geq 64(n=249)$ \\
\hline \multicolumn{5}{|l|}{ High creatinine } \\
\hline Continuous, hr/day & $0.997(0.629-1.581)$ & $1.075(0.791-1.461)$ & $1.097(0.646-1.863)$ & $1.074(0.747-1.545)$ \\
\hline \multicolumn{5}{|l|}{ Categorical, hr/day } \\
\hline$<7$ & $0.754(0.197-2.889)$ & $0.376(0.115-1.227)$ & $0.336(0.064-1.770)$ & $0.333(0.079-1.407)$ \\
\hline 7 to $<8$ & 1.000 & 1.000 & 1.000 & 1.000 \\
\hline$\geq 8$ & $0.980(0.255-3.771)$ & $0.713(0.297-1.712)$ & $0.711(0.137-3.696)$ & $0.724(0.233-2.246)$ \\
\hline \multicolumn{5}{|l|}{ Low eGFR } \\
\hline Continuous, hr/day ${ }^{\mathrm{b}}$ & $1.135(0.689-1.871)$ & $1.086(0.825-1.429)$ & $1.330(0.757-2.336)$ & $1.134(0.816-1.577)$ \\
\hline \multicolumn{5}{|l|}{ Categorical, hr/day } \\
\hline$<7$ & $0.707(0.154-3.239)$ & $0.576(0.218-1.523)$ & $0.417(0.074-2.336)$ & $0.540(0.150-1.937)$ \\
\hline 7 to $<8$ & 1.000 & 1.000 & 1.000 & 1.000 \\
\hline$\geq 8$ & $1.238(0.300-5.104)$ & $0.782(0.349-1.750)$ & $1.129(0.233-5.465)$ & $0.860(0.292-2.534)$ \\
\hline \multicolumn{5}{|l|}{ CKD } \\
\hline Continuous, hr/day ${ }^{b}$ & $1.083(0.683-1.718)$ & $1.095(0.842-1.424)$ & $1.259(0.721-2.199)$ & $1.075(0.787-1.469)$ \\
\hline \multicolumn{5}{|l|}{ Categorical, hr/day } \\
\hline$<7$ & $0.754(0.197-2.889)$ & $0.616\left(0.244-1.55^{8}\right)$ & $0.514\left(0.105^{-2.522}\right)$ & $0.608(0.186-1.988)$ \\
\hline 7 to $<8$ & 1.000 & 1.000 & 1.000 & 1.000 \\
\hline$\geq 8$ & $0.980(0.255-3.771)$ & $0.841(0.388-1.823)$ & $0.921(0.194-4.367)$ & $0.799(0.289-2.211)$ \\
\hline
\end{tabular}

CKD, chronic kidney disease; OR, odds ratio; CI, confidence interval; eGFR, estimated glomerular filtration rate.

${ }^{a}$ Adjusted model: adjusted for sex, age, body mass index, systolic blood pressure, smoking, alcohol, exercise, diabetes mellitus, hypercholesterolemia, depression, history of cardiovascular disease, history of cancer, menopause, socioeconomic status (household income, marital status, and working status), and sleep quality (sleep difficulty, sleep awakeness, snoring, and sleep apnea).

${ }^{\mathrm{b}}$ Continuous model: association analysis on total sleep duration as a continuous variable. 
Supplementary Table 3. Age-specific association between sleep duration and CKD criteria in women $(\mathbf{n}=816)$

\begin{tabular}{|c|c|c|c|c|}
\hline \multirow{2}{*}{ Sleep duration } & \multicolumn{2}{|c|}{ Unadjusted OR (95\% CI) } & \multicolumn{2}{|c|}{ Adjusted OR $(95 \% \text { CI })^{\mathrm{a}}$} \\
\hline & Age $<60(n=423)$ & Age $\geq 60(n=393)$ & Age $<60(n=423)$ & Age $\geq 60(n=393)$ \\
\hline \multicolumn{5}{|l|}{ High creatinine } \\
\hline Continuous, hr/day ${ }^{\mathrm{b}}$ & $1.337(0.899-1.988)$ & $1.258(0.995-1.592)$ & $1.277(0.837-1.946)$ & $1.224(0.932-1.607)$ \\
\hline \multicolumn{5}{|l|}{ Categorical, hr/day } \\
\hline$<7$ & $0.938(0.280-3.144)$ & $2.076(0.752-5.734)$ & $0.796(0.213-2.979)$ & $2.121(0.627-7.179)$ \\
\hline 7 to $<8$ & 1.000 & 1.000 & 1.000 & 1.000 \\
\hline$\geq 8$ & $1.503(0.492-4.590)$ & $3.182(1.256-8.059)$ & $1.289(0.376-4.416)$ & $3.174(1.030-9.785)$ \\
\hline \multicolumn{5}{|l|}{ Low eGFR } \\
\hline Continuous, hr/day ${ }^{b}$ & $1.461(0.955-2.235)$ & $1.197(0.970-1.476)$ & $1.469(0.885-2.441)$ & $1.184(0.926-1.513)$ \\
\hline \multicolumn{5}{|l|}{ Categorical, hr/day } \\
\hline$<7$ & $1.132(0.278-4.615)$ & $1.866(0.789-4.413)$ & $1.038(0.221-4.879)$ & $1.741(0.627-4.832)$ \\
\hline 7 to $<8$ & 1.000 & 1.000 & 1.000 & 1.000 \\
\hline$\geq 8$ & $2.284(0.653-7.985)$ & $2.587(1.174-5.703)$ & $2.181(0.528-9.004)$ & $2.597(1.018-6.627)$ \\
\hline \multicolumn{5}{|l|}{ CKD } \\
\hline Continuous, hr/day ${ }^{b}$ & $1.479(1.000-2.185)$ & $1.210(0.982-1.491)$ & $1.581(0.969-2.577)$ & $1.205(0.945-1.537)$ \\
\hline \multicolumn{5}{|l|}{ Categorical, hr/day } \\
\hline$<7$ & $0.900(0.237-3.420)$ & $1.866(0.789-4.413)$ & $0.852(0.196-3.704)$ & $1.692(0.613-4.673)$ \\
\hline 7 to $<8$ & 1.000 & 1.000 & 1.000 & 1.000 \\
\hline$\geq 8$ & $2.374(0.775-7.273)$ & $2.697(1.227-5.929)$ & $2.471(0.687-8.886)$ & $2.699(1.065-6.841)$ \\
\hline
\end{tabular}

CKD, chronic kidney disease; OR, odds ratio; CI, confidence interval; eGFR, estimated glomerular filtration rate.

aAdjusted model: adjusted for sex, age, body mass index, systolic blood pressure, smoking, alcohol, exercise, diabetes mellitus, hypercholesterolemia, depression, history of cardiovascular disease, history of cancer, menopause, socioeconomic status (household income, marital status, and working status), and sleep quality (sleep difficulty, sleep awakeness, snoring, and sleep apnea).

${ }^{\mathrm{b}}$ Continuous model: association analysis on total sleep duration as a continuous variable. 
Supplementary Table 4. Association between sleep duration and CKD criteria after excluding hypertension, diabetes mellitus, and cardiovascular disease

\begin{tabular}{|c|c|c|c|c|}
\hline \multirow{2}{*}{ Exclusion criteria } & \multirow{2}{*}{ Total no. } & \multirow{2}{*}{ No. (\%) } & \multicolumn{2}{|c|}{ OR, hr/day ${ }^{\mathrm{a}}$} \\
\hline & & & Unadjusted OR (95\% CI) & Adjusted OR $(95 \% \mathrm{CI})^{\mathrm{b}}$ \\
\hline \multicolumn{5}{|l|}{ High creatinine } \\
\hline \multicolumn{5}{|l|}{ Hypertension } \\
\hline Men & 332 & $14(4.2)$ & $1.186(0.770-1.829)$ & $1.345(0.764-2.370)$ \\
\hline Women & 512 & $16(3.1)$ & $1.196(0.792-1.806)$ & $1.246(0.697-2.228)$ \\
\hline \multicolumn{5}{|l|}{ Diabetes mellitus } \\
\hline Men & 460 & $34(7.4)$ & $1.168(0.882-1.547)$ & $1.154(0.837-1.591)$ \\
\hline Women & 735 & $46(6.3)$ & $1.239(0.979-1.569)$ & $1.127(0.880-1.444)$ \\
\hline \multicolumn{5}{|c|}{ Cardiovascular disease } \\
\hline Men & 485 & $31(6.4)$ & $0.996(0.751-1.321)$ & $1.012(0.745-1.373)$ \\
\hline Women & 772 & $49(6.4)$ & $1.289(1.029-1.614)$ & $1.264(1.004-1.592)$ \\
\hline \multicolumn{5}{|l|}{ Low eGFR } \\
\hline \multicolumn{5}{|l|}{ Hypertension } \\
\hline Men & 332 & $16(4.8)$ & $1.076(0.716-1.618)$ & $1.172(0.686-2.004)$ \\
\hline Women & 512 & $17(3 \cdot 3)$ & $1.249(0.838-1.860)$ & $1.285(0.701-2.357)$ \\
\hline \multicolumn{5}{|l|}{ Diabetes mellitus } \\
\hline Men & 460 & $37(8.0)$ & $1.178(0.899-1.544)$ & $1.184(0.867-1.617)$ \\
\hline Women & 735 & $55(7 \cdot 5)$ & $1.239(0.997-1.540)$ & $1.110(0.883-1.397)$ \\
\hline \multicolumn{5}{|c|}{ Cardiovascular disease } \\
\hline Men & 485 & $34(7.0)$ & $1.117(0.853-1.462)$ & $1.129(0.845-1.508)$ \\
\hline Women & 772 & $57(7 \cdot 4)$ & $1.247(1.010-1.539)$ & $1.213(0.975-1.509)$ \\
\hline \multicolumn{5}{|l|}{ Low CKD } \\
\hline \multicolumn{5}{|l|}{ Hypertension } \\
\hline Men & 332 & $18(5 \cdot 4)$ & $1.119(0.761-1.645)$ & $1.127(0.705-1.800)$ \\
\hline Women & 512 & $18(3.5)$ & $1.224(0.829-1.805)$ & $1.267(0.720-2.229)$ \\
\hline \multicolumn{5}{|l|}{ Diabetes mellitus } \\
\hline Men & 460 & $39(8.5)$ & $1.153(0.885-1.501)$ & $1.136(0.836-1.545)$ \\
\hline Women & 735 & $58(7 \cdot 9)$ & $1.248(1.009-1.543)$ & $1.144(0.913-1.432)$ \\
\hline \multicolumn{5}{|c|}{ Cardiovascular disease } \\
\hline Men & 485 & $38(7.8)$ & $1.083(0.838-1.399)$ & $1.064(0.807-1.402)$ \\
\hline Women & 772 & $61(7 \cdot 9)$ & $1.269(1.035-1.557)$ & $1.237(1.000-1.530)$ \\
\hline
\end{tabular}

CKD, chronic kidney disease; OR, odds ratio; CI, confidence interval; eGFR, estimated glomerular filtration rate. ${ }^{\mathrm{a}}$ Continuous model: association analysis on total sleep duration as a continuous variable.

bAdjusted model: adjusted for sex, age, body mass index, systolic blood pressure, smoking, alcohol, exercise, diabetes mellitus, hypercholesterolemia, depression, history of cancer, menopause, socioeconomic status (household income, marital status, and working status), and sleep quality (sleep difficulty, sleep awakeness, snoring, and sleep apnea). 


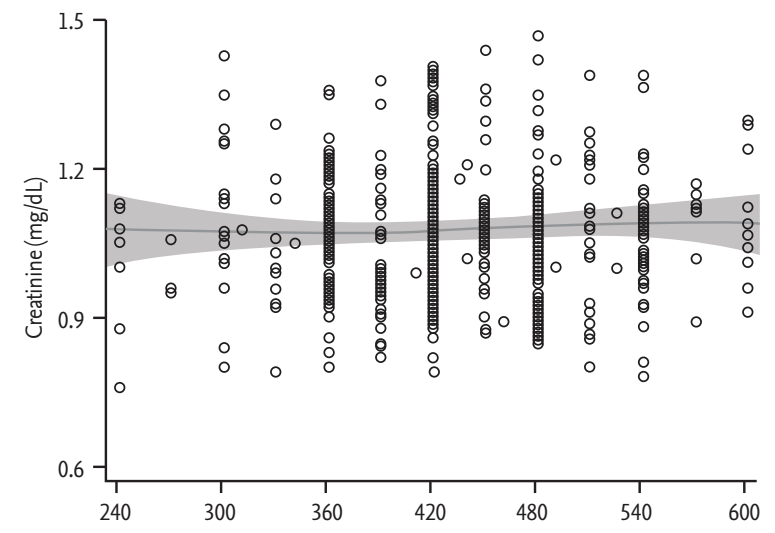

A

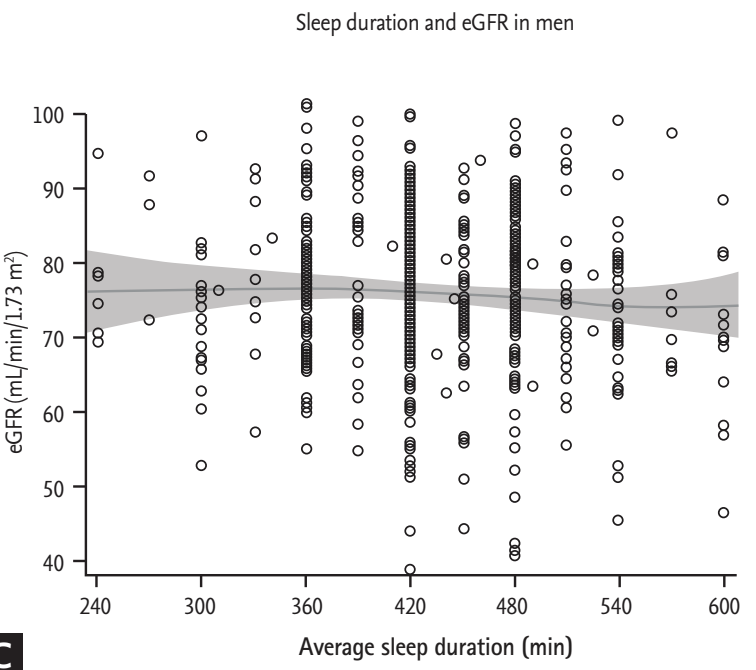

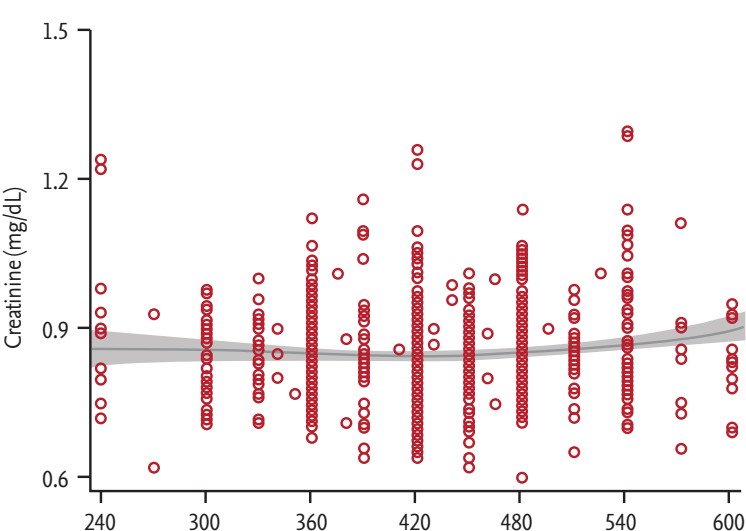

B

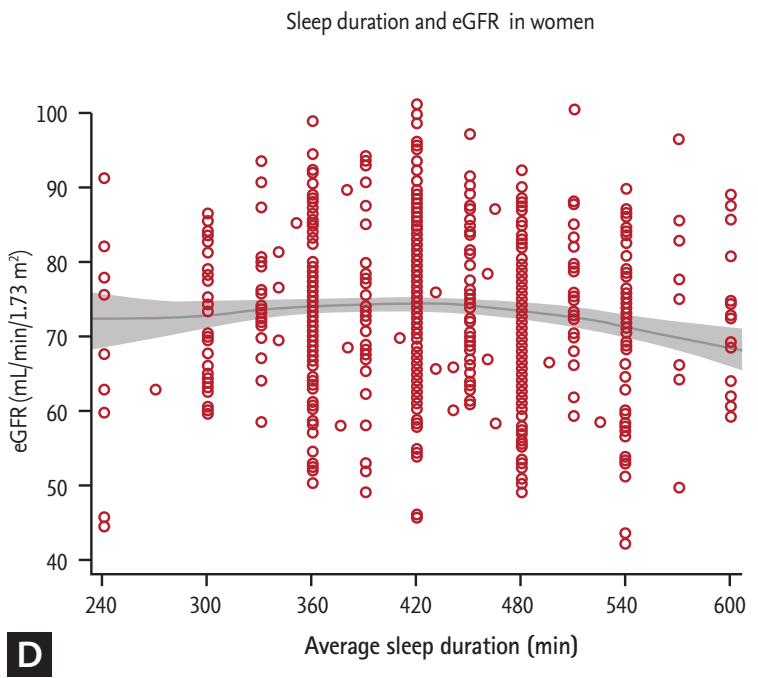

D

Supplementary Figure 1. Cubic spline functions of the association between average sleep duration and kidney function according to sex. (A) Cubic spline functions of the association between average sleep duration and creatinine in men. (B) Cubic spline functions of the association between average sleep duration and creatinine in women. (C) Cubic spline functions of the association between average sleep duration and estimated glomerular filtration rate (eGFR) in men. (D) Cubic spline functions of the association between average sleep duration and eGFR in women. The shadowed areas represent $95 \%$ confidence intervals for the fitted splines. The solid line is a restricted cubic spline. The black dots represent men and the red dots women. 